\title{
A RANDOM MAP MODEL FOR QUANTUM INTERFERENCE
}

\author{
ABRAHAM BOYARSKY AND PAWEŁ GÓRA
}

\begin{abstract}
Quantum interference of particle systems results from the wave properties of the particles and are predicted theoretically from the superposition of the wave functions. In place of wave functions we use deterministic chaotic maps as the underlying mechanism that produces the observed probability density functions. Let $\psi_{i}(x, t), i=1,2$ be two wave functions of a quantum mechanical particle system. For each $\psi_{i}(x, t)$ we define deterministic nonlinear point transformations $\tau_{i}(x)$ whose unique probability density function is the observed density $\rho_{i}(x, t)=\psi_{i}^{*}(x, t) \psi_{i}(x, t)$. We consider the wave function $\psi(x, t)=$ $a \psi_{1}(x, t)+b \psi_{2}(x, t)$ and show that we can associate with $\psi(x, t)$, a random chaotic map that switches (probabilistically between) $\tau_{1}(x)$, $\tau_{2}(x)$ and the identity map $I(x)$ and whose probability density function $f_{t}(x)$ equals $\psi^{*}(x, t) \psi(x, t)$, where $t$ denotes time. This description of quantum interference of particle systems allows a more insightful interpretation than wave mechanics.

Keywords: Wave function, deterministic chaotic transformation, position dependent random map, two-slit experiment.

\section{Introduction}

Chaotic maps are deterministic yet behave statistically asymptotically in time. In this note we present a chaotic dynamical model that reproduces the results of quantum interference experiments such as the two-slit experiment. In place of wave functions we shall use real chaotic maps as the underlying mechanism for the observed probability density functions. Let $\psi_{i}(x, t), i=1,2$ be two wave functions of a quantum mechanical particle system. We associate with each $\psi_{i}(x, t)$ a deterministic nonlinear point transformation $\tau_{i}(x)$ whose unique invariant probability density function is the observed density $\rho_{i}(x, t)=\psi_{i}^{*}(x, t) \psi_{i}(x, t)$. We consider the superposition wave function $\psi(x, t)=a \psi_{1}(x, t)+b \psi_{2}(x, t)$ and show that we can associate with $\psi(x, t)$, a random chaotic map related to $\tau_{1}(x)$ and $\tau_{2}(x)$, whose
\end{abstract}

The research has been supported by NSERC grants. 
probability density function $f_{t}(x)$ is equal to $\psi^{*}(x, t) \psi(x, t)$, where $t$ denotes time. The key result is that random switching between the maps $\tau_{1}(x)$ and $\tau_{2}(x)$ and the identity map $I(x)$ accomplishes the same as the phases of the wave functions. However, the random map description allows a physical and insightful interpretation of quantum interference by using particle trajectories. In Section 2 we present the notation and a review of chaotic maps. In Section 3, we review the motivation for our discrete time model for quantum mechanics. In Section 4, we use a result from [3] to associate a position dependent random map to the superposition of wave functions and identify the relationship with the individual wave functions. This identification is reversible so that given any random map that describes interference, we can identify the wave functions which would produce the interference using conventional quantum mechanics. In Section 5 numerical simulation of a two-slit experiment is done which shows that the random map dynamics achieves the interference pattern accurately.

\section{Notation and Review}

Let $\mathrm{R}=(-\infty, \infty)$ and let $T: \mathbb{R} \rightarrow \mathbb{R}$ possess a unique absolutely continuous invariant measure $\mu$ which has the probability density function (pdf) $f$, that is

$$
\int_{A} f d x=\int_{T^{-1} A} f d x
$$

for any measurable set $A \subset \mathbb{R}$. The Frobenius-Perron $P_{T} f$ operator acting on the space of integrable functions is defined by

$$
\int_{T^{-1} A} f d x=\int_{A} P_{T} f d x
$$

The operator $P_{T}$ transforms probability density functions into probability density functions under the transformation $T$, where $T$ is assumed to be nonsingular.

Let $h: \mathbb{R} \rightarrow \mathbb{R}$ be a diffeomorphism. Then $\tau=h \circ T \circ h^{-1}$ is a transformation from $\mathbb{R}$ into $\mathbb{R}$ which is said to be differentially conjugate to $T$ and whose probability density function is given by

$$
k=\left(f \circ h^{-1}\right) \cdot\left|\left(h^{-1}\right)^{\prime}\right|
$$

Let the transformation $T$ possess the probability density function $f$. Suppose we are given a probability density function $g$ on $\mathbb{R}$, can we find a transformation $\tau$, derived from $T$, such that $g$ is the unique 
probability density function invariant under $\tau$ ? The answer is yes. Using (1), we must find $h^{-1}$ such that

$$
\left(f \circ h^{-1}\right) \cdot\left(h^{-1}\right)^{\prime}=g,
$$

where we have assumed, without loss of generality, that $h^{-1}$ is an increasing function on $\mathbb{R}$. Now let

$$
F(x)=\int_{-\infty}^{x} f(y) d y
$$

be the distribution function associated with $f$. Then, from (5) and the change of variable formula, we have

$$
F\left(h^{-1}(x)\right)=\int_{-\infty}^{x} g(y) d y
$$

Since $F$ is a monotonically increasing function, it has a unique inverse and

$$
h^{-1}(x)=F^{-1}\left(\int_{-\infty}^{x} g(y) d y\right)
$$

Thus, we have found $h^{-1}(x)$ and hence $h(x)$ such that $\tau=h \circ$ $T \circ h^{-1}$ has the unique probability density function $g(x)$. Summarizing, given any probability density function $g(x)$, we have shown the existence of a point transformation $\tau$ whose unique probability density function is $g(x)$.

3. Chaotic Dynamical System Model for Quantum Interference

We make two general assumptions for our model:

1) Time is discrete, as implied by string theory and quantum loop gravity. This, of course, implies that all continuous time theories such as quantum mechanics and general relativity are at best only good approximations to a discrete time reality.

2) Observables such as position and velocity are described by probability density functions. In classical mechanics and relativity theory, we can view the flows as point measures rather than points, which may be approximations to pdfs with very narrow support on $R^{3}$.

Quantum mechanics formalism stipulates that the absolute value of the wave function, $\psi$, is the observable pdf, $f$. In our model of the underlying process for particle motion, we use a real chaotic map, $\tau$, that generates $f$, via the Birkhoff Ergodic Theorem. We postulate that the iteration time is of the order of the Planck time, $10^{-44}$ seconds, while the observation time is of the order of $10^{-10}$ seconds or more. The gap in these times allows for many iterations of $\tau$ between observations and so, by the Birkhoff Ergodic Theorem, reveals the observable pdf. 
Now let $\left\{\tau_{1}, \tau_{2, \ldots \ldots,}, \tau_{K}\right\}$ be a collection of 1-dimensional maps and define a random map to be a discrete-time dynamical system in which one of the maps is randomly selected and applied at each iteration with constant probability $p_{k}, p_{k}>0, \sum_{k=1}^{K} p_{k}=1$. A measure $\mu$ on $[0,1]$ is called invariant under $\tau$ if

$$
\mu(A)=\sum_{k=1}^{K} p_{k} \mu\left(\tau_{k}^{-1} A\right)
$$

for each measurable set A. In [2] it is shown that the following sufficient condition is sufficient for the existence of an absolutely continuous invariant measure for such a random map:

$$
\sum_{k=1}^{K} \frac{p_{k}}{\left|\tau_{k}^{\prime}\right|} \leq \gamma<1
$$

for some constant $\gamma$.

Although such dynamical systems have application in the study of fractals [4] they are not rich enough for our purposes because they do not generate a sufficiently large class of invariant densities. To enlarge the class of pdfs that are attainable from random maps we allow the probabilities of selecting the maps to be functions of position. The main result of [3] provides a sufficient condition for the existence of an absolutely continuous invariant measure for position dependent random maps. The pdf $f(x)$ of this measure is the solution of the equation:

$$
\sum_{k=1}^{K} P_{\tau_{k}}\left(p_{k}(x) f(x)\right)=f(x)
$$

where $P_{\tau_{k}}$ is the Frobenius-Perron operator associated with $\tau_{k}$. If $\Gamma=$ $\left\{\tau_{1}, \ldots, \tau_{K}\right\}$ is a set of maps, we denote by $A_{\Gamma}$ the set of all attainable densities, i.e., the set of densities $f$ which satisfy (6), for all possible choices of probability weight $\left\{p_{1}(x), \ldots, p_{K}(x)\right\}$. We now state the result of [3] that is needed in the sequel.

Proposition 1. If the set of maps $\Gamma=\left\{\tau_{1}, \ldots, \tau_{K}\right\}$ contains the identity map, then the set $A_{\Gamma}$ of attainable probability densities is equal to the set of all probability densities.

Proof. The identity map preserves every probability density function. 
We will use the following theorem from [3].

Theorem 2. Let $\left\{\tau_{1}, \ldots, \tau_{K}\right\}$ be a collection of maps. Let $f_{k}$ be a probability density function of $\tau_{k}, k=1, \ldots, K$. For any positive constants $a_{k}, k=1, \ldots, K$, there exists a system of probability functions $p_{1}, \ldots, p_{K}$ such that the density $f=a_{1} f_{1}+\cdots+a_{K} f_{K}$ is invariant under the random map $T=\left\{\tau_{1}, \ldots, \tau_{K} ; p_{1}, \ldots, p_{K}\right\}$. It is enough to set

$$
p_{k}=\frac{a_{k} f_{k}}{a_{1} f_{1}+\cdots+a_{K} f_{K}} \quad, \quad k=1,2, \ldots, K,
$$

where we assume that $0 / 0=0$.

\section{Quantum Interference}

Let $\psi_{1}$ and $\psi_{2}$ be two wave functions. By the foregoing method, we can construct point transformations $\tau_{1}$ and $\tau_{2}$ whose probability density functions 's are $\psi_{1}^{*} \psi_{1}$ and $\psi_{2}^{*} \psi_{2}$, respectively. Interference is the result of addition (superposition) of these wave functions, that is, $\psi=a \psi_{1}+$ $b \psi_{2}$. Clearly $\psi^{*} \psi$ is a probability density function which, in general, is time dependent. By Proposition 1, we know that $\psi^{*} \psi$ can be realized at any time $t$ as a position dependent random map $T=\left\{\tau_{1}, \tau_{2}, I\right.$; $\left.p_{1}(x), p_{2}(x), 1-p_{1}(x)-p_{2}(x)\right\}$ where the weighting probabilities $p_{1}(x)$, $p_{2}(x)$ and hence $1-p_{1}(x)-p_{2}(x)$ are determined by Theorem 2 . Note that whereas $\tau_{1}, \tau_{2}$ represent particle motion under the influence of one slit or the other alone such as in the two-slit experiment, the identity function $I$ represents a waiting effect, where the particle may move but during the iteration time remains in the same local position

We now ask: can this procedure be reversed. That is, given density functions $f_{1}(x), f_{2}(x), f_{t}(x)$, can we find wave functions $\psi_{1}$ and $\psi_{2}$ such that $f_{1}=\psi_{1}^{*} \psi_{1}, f_{2}=\psi_{2}^{*} \psi_{2}$ and $f_{t}(x)=\left(\psi_{1}+\psi_{2}\right)^{*}\left(\psi_{1}+\psi_{2}\right)$. The answer is yes. Let $\psi_{1}=\sqrt{f_{1}} e^{i s_{1} t}$ and $\psi_{2}=\sqrt{f_{2}} e^{i s_{2} t}$. Then

$$
\left(\psi_{1}+\psi_{2}\right)^{*}\left(\psi_{1}+\psi_{2}\right)=f_{1}+f_{2}+2 \sqrt{f_{1} f_{2}}\left[\cos \left(s_{1}-s_{2}\right) t\right]=f_{t}
$$

Since $f_{1}, f_{2}$ and $f_{t}$ are known, $\left(s_{1}-s_{2}\right)$ can be computed from (7). Thus, we have determined $\psi_{1}$ and $\psi_{2}$ up to a constant phase.

We summarize the above in our main result:

Theorem 3. Let $\psi_{1}$ and $\psi_{2}$ be two wave functions. We can construct point transformations $\tau_{1}$ and $\tau_{2}$ whose density functions are $\psi_{1}^{*} \psi_{1}$ and $\psi_{2}^{*} \psi_{2}$, respectively. The density function realized from the superposed wave function $\psi=a \psi_{1}+b \psi_{2}$ can be achieved by a random map based on $\tau_{1}, \tau_{2}$ and I with weighting probabilities that are determined from $\psi_{1}$ and $\psi_{2}$ as in Theorem 2. Starting with observed density functions $f_{1}, f_{2}$ and $f_{t}$, the process can be reversed and wave functions $\psi_{1}$ and $\psi_{2}$ determined. 


\section{Example: Two-slit experiment}

Let us consider the two-slit experiment, with slit size .01 units and slit centers located at positions $x=-1$ and $x=1$. We assume that the pdf's at the slits are Gaussian densities with variance .005 as shown in Figures 1a) and $1 \mathrm{~b}$ ), that is,

$$
\begin{aligned}
& f_{1}(x)=\frac{1}{10 \sqrt{\pi}} \exp \left(-(x+1)^{2} / 100\right), \\
& f_{2}(x)=\frac{1}{10 \sqrt{\pi}} \exp \left(-(x-1)^{2} / 100\right),
\end{aligned}
$$

and their superposition is given by equation (24) of [5] which, for $t=1$, becomes

$$
\begin{aligned}
f(x)=\frac{1}{2+\exp (-1 / 100-100)}[ & f_{1}(x)+f_{2}(x) \\
& \left.+\frac{1}{10 \sqrt{\pi}} \cos (2 x) \exp \left(-\left(x^{2}+1\right) / 100\right)\right] .
\end{aligned}
$$

The random map we now construct consists of three maps, $\tau_{i}, i=1,2$, constructed as in Section 2 which have pdfs $f_{1}$ and $f_{2}$, respectively, and the identity map $\tau_{3}(x)=x$. We can view this random map as giving the particle a choice of moving to the left (under the influence of the left slit), moving to the right (under the influence of the right slit) or remaining in the same place, as reflected in the identity map $\tau_{3}$. If the iteration time is some multiple of say the Planck time, then within an iteration time a particle can move left and right a number of times and still at the iteration time end up close to its original position.

It can be easily shown that if we set $a_{1}=a_{2}=0.1, a_{3}=0.8$, then

$$
f_{3}(x)=\frac{1}{a_{3}}\left[f(x)-f_{1}(x)-f_{2}(x)\right],
$$

is a density and the superposition density can be written as

$$
f=a_{1} f_{1}+a_{2} f_{2}+a_{3} f_{3} .
$$

They are shown in Figures 1 and 2. Figure 1 shows $f_{1}$ in part a) and $f_{2}$ in b). Figure 2 shows $f$ in a) and $f_{3}$ in b).

According to Theorem 2 we construct the probabilities

$$
p_{i}(x)=\frac{a_{i} f_{i}(x)}{f(x)}, \quad i=1,2,3 .
$$

They are shown in Figure 3. 

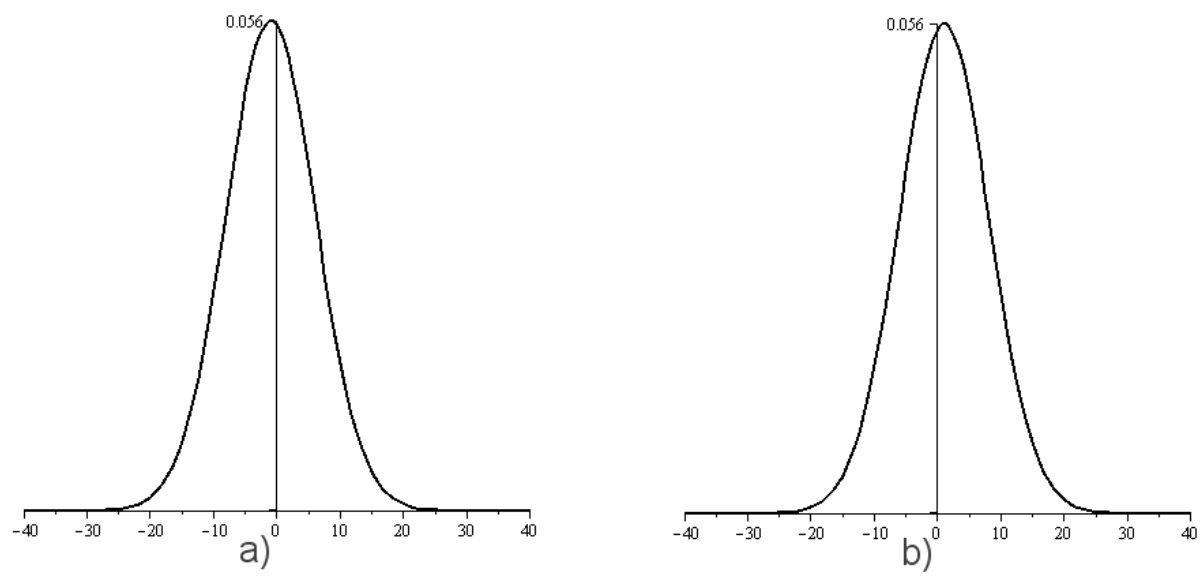

Figure 1. a) shows density $f_{1}$, b) shows density $f_{2}$.
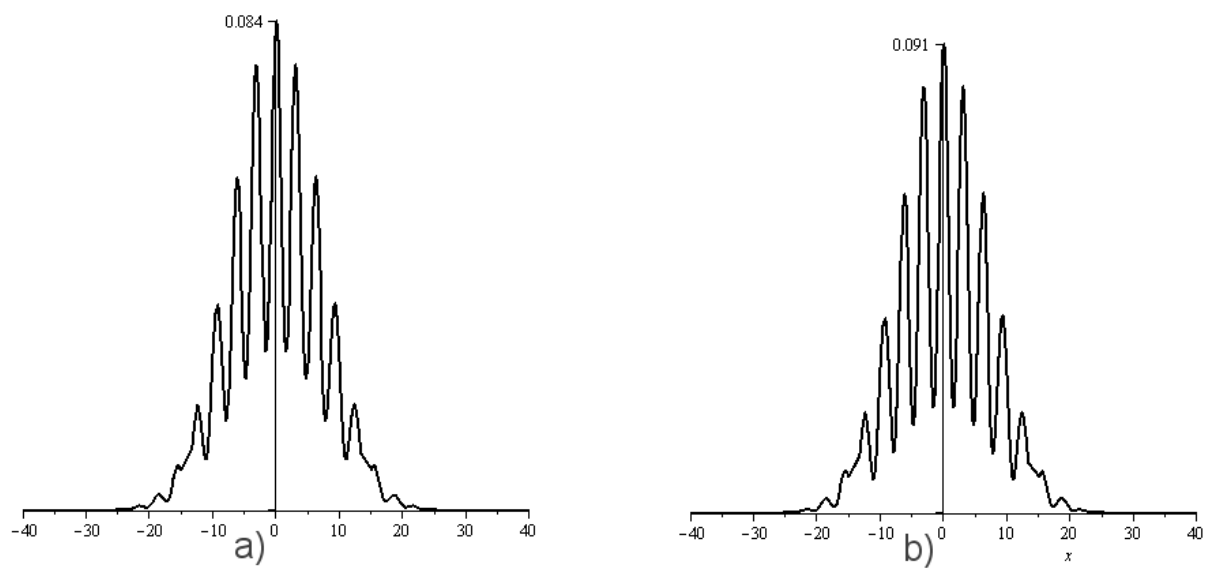

Figure 2. a) shows density $f$, b) shows density $f_{3}$.

Using the "recipe" from Section 2 we construct maps $\tau_{1}$ and $\tau_{2}$ corresponding to the densities $f_{1}$ and $f_{2}$. We have

$$
h_{1}^{-1}(x)=\int_{-\infty}^{x} f_{1}(t) d t=\frac{1}{2}+\frac{1}{2} \operatorname{erf}\left(\frac{1}{10} x+\frac{1}{10}\right)
$$

and

$$
h_{2}^{-1}(x)=\int_{-\infty}^{x} f_{2}(t) d t=\frac{1}{2}+\frac{1}{2} \operatorname{erf}\left(\frac{1}{10} x-\frac{1}{10}\right)
$$

where

$$
\operatorname{erf}(x)=\frac{2}{\sqrt{\pi}} \int_{0}^{x} \exp \left(-t^{2}\right) d t
$$




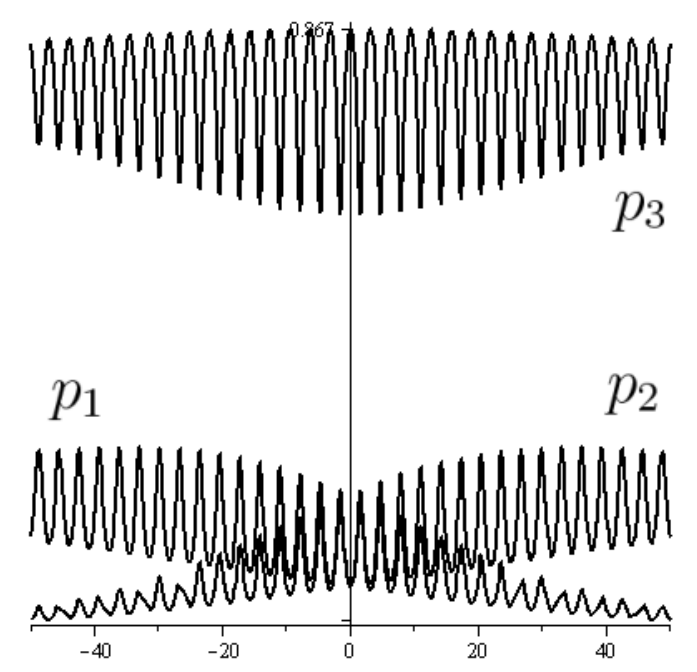

Figure 3. The position dependent probabilities $p_{1}, p_{2}, p_{3}$.

We define $\tau_{i}=h_{i} \circ T \circ h_{i}^{-1}, i=1,2$, where $T(x)=1-2|x-1 / 2|$ is the tent map. We used $\tau_{3}(x)=x$ as the third map. Figure 4 shows the maps $\tau_{1}$ (left) and $\tau_{2}$ (right) in part a) and in part b) the results of numerical simulation of 2,000,000 iterations of random map $\left\{\tau_{1}, \tau_{2}, \tau_{3} ; p_{1}, p_{2}, p_{3}\right\}$.
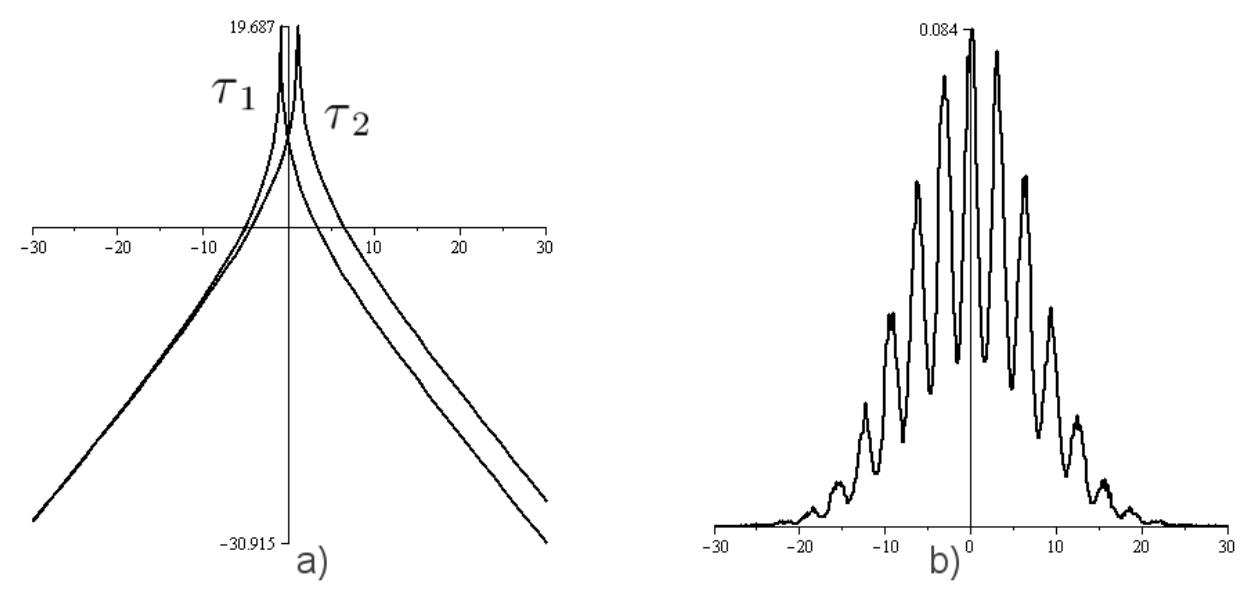

Figure 4. a) maps $\tau_{1}$ and $\tau_{2}$ b) results of numerical simulation.

\section{Observations:}

1) In the foregoing model of the 2-slit experiment we are not compelled to say that the particle goes through both slits. Rather, we say that the particle is a particle and passes through one slit or the other. 
But between the slit screen and the detecting screen the particles motion is governed by the spacetime geometry determined by the physical structure of the experiment (size of 2 open slits, their separation and distance between slit screen and detecting screen) and which is described mathematically by the weighting probabilities for the 2 maps in the random map. From this perspective spacetime in the quantum setting is a complex structure which can be described probabilistically.

2) The random transformation model for quantum interference lends itself to an interpretation of nonlocality since a jump from one transformation to another is a discontinuous effect that can propel a quantum particle across the universe in the time span of one iteration of the process.

3) Since the map $\tau_{i}$ is piecewise onto all of the real line $R$, a few, iterations amount to a very small duration of time, but during this time the particle orbit may traverse a large part of $R$. Also, the switching from one map to another can cause the particle to be pushed far out. This process, iterating at the Planck time, may explain nonlocality since the particle may appear to be in two distant locations at once during the observation time which is many times as large as the Planck time, and hence during such an observation the particle may have the time to travel back and forth between the two positions numerous times, at (finite) speeds far greater than that of light.

4) The choice of the maps $\tau_{i}$ may not be unique. However this would not change the foregoing theory since all we need is a dynamical mechanism that generates the desired pdfs. How fast or slow this is accomplished is not important since the physical process is assumed to have a very small iteration time.

\section{REFERENCES}

[1] A. Boyarsky and P. Góra A Description of Stochastic Systems Using Chaotic Maps, J. Appl. Math. Stoch. Anal. 2004:2 (2004), 137-141.

[2] S. Pelikan, Invariant densities for random maps of the interval, Trans. Amer. Math Soc. 281 (1984), 813-824.

[3] P. Góra, and A. Boyarsky, Absolutely continuous invariant measures for random maps with position dependent probabilities, J. Math. Anal. Appl. 278 (2003), $225-242$.

[4] M. Barnsley, Fractals Everywhere, Academic Press, London, 1988.

[5] M. McClendon and H. Rabitz, Numerical simulations in stochastic mechanics, Physical Review A 37, No. 9, (1998), 3479-3492.

Department of Mathematics and Statistics,, Concordia University 7141 Sherbrooke Street West,, Montreal, Quebec H4B 1R6, Canada, E-MAIL: BOYAR@ALCOR.CONCORDIA.CA,,PGORA@MATHSTAT.CONCORDIA.CA, TEL. 514-848-2424, EXT. 3240, FAX 514-848-2831 